\title{
Enfoque territorial y los Objetivos de Desarrollo Sostenible: Programas de Desarrollo con Enfoque Territorial en Colombia y su papel en la sostenibilidad social
}

\author{
Territorial Approach and Sustainable Development \\ Objectives: Development Programs with territorial \\ approach in Colombia and their role in social sustainability
}

\author{
Diego Alejandro Álvarez Pinzón* \\ Angie Paola Mendoza Alfonso** \\ Jhon Alexander Idrobo-Velasco*** \\ Jorge Andrés Pinzón Rueda**** \\ Artículo de reflexión
}

Fecha de recepción: 31 de mayo de 2021

Fecha de aceptación: 21 de julio de 2021

\section{Para citar este artículo:}

Álvarez Pinzón, D. A., Mendoza Alfonso, A. P., Idrobo-Velasco J. A. y Pinzón Rueda, J. A. (2021). Enfoque territorial y los Objetivos de Desarrollo Sostenible: Programas de Desarrollo con Enfoque Territorial en Colombia y su papel en la sostenibilidad social. Revista Análisis Jurídico-Político, 3(6), 147-168. https://doi.org/10.22490/26655489.4782

\footnotetext{
* Estudiante de la Facultad de Sociología de la Universidad Santo Tomás, integrante del Semillero de investigación EIRENE Latinoamérica y miembro del grupo de investigación Estudios Interdisciplinarios de la Sociedad y la Cultura, líneas de investigación en construcción de paz territorial. Correo electrónico: diegoalvarezp@usantotomas.edu.co. Orcid: 0000-0001-5256-3962

** Estudiante de la Facultad de Sociología de la Universidad Santo Tomás, partícipe en proyectos de investigación en el marco de la paz y los escenarios de posconflictos en la convocatoria de Jóvenes Investigadores 2020. Integrante del semillero de Sociedad y Consumo. Correo electrónico: angie.mendoza@usantotomas.edu.co. Orcid: 0000-0002-3393-8738

*** Licenciado en Filosofía y Magíster en Filosofía latinoamericana por la Universidad Santo Tomás. Docente-investigador de la Facultad de Sociología de la Universidad Santo Tomás-Bogotá. Líder del Grupo de investigación interinstitucional Tlamatinime y coordinador del Semillero de Investigación Eirene Latinoamérica. Correo electrónico: jhonidrobo@usantotomas.edu.co. Orcid: 0000-0002-2228-0002. Q.E.P.D

$* * * *$ Especialista en instrumentos de ordenamiento urbano-regional y Magister en urbanismo de la Universidad Nacional de Colombia. Docente de la Facultad de Sociología de la Universidad Santo Tomás-Bogotá. Correo electrónico: jorgepinzonr@usantotomas.edu.co. ORCID: 0000-0002-0519-4524
} 


\section{Resumen}

Este artículo busca evidenciar la importancia de la posible relación entre los PDET (Programas de Desarrollo con Enfoque Territorial) y los ODS (Objetivos de Desarrollo Sostenible) como una estrategia para promover la sostenibilidad social, que permita la transformación y la renovación de los territorios en Colombia, dentro del marco de la construcción de paz, además de fortalecer las políticas públicas participativas con un enfoque botton-up, con el fin de articular los diferentes actores presentes en los territorios.

Se presenta en un primer momento las generalidades de los ODS y su agenda mundial 2030. En un segundo apartado se explican los PDET en el marco del Acuerdo de Paz en Colombia. En un tercer momento se busca generar una reflexión sobre la armonización entre los ODS y los PDET, lo que permite reconocer puntos de encuentro y diferencias, como una estrategia que promueva la sostenibilidad social. En el cuarto apartado de este artículo se discutirá sobre el diseño de políticas públicas participativas desde un enfoque bottom-up que permita aportar a la construcción de paz, ya que los PDET y los ODS están diseñados en clave de esta participación, lo que busca responder de manera efectiva a las necesidades de las comunidades y su entorno. Finalmente, se presentan las conclusiones que evidencian cómo los PDET con los ODS podrían articularse para la transformación y renovación de los territorios en Colombia.

Palabras clave: PDET, ODS, territorio, posconflicto en Colombia, sostenibilidad social.

\section{Abstrac}

This article seeks to highlight the importance of the possible relationship between the PDET and the SDGs, as a strategy to promote social sustainability that allows transformation and renewal of territories in Colombia in the framework of peacebuilding. In addition, to strengthen public policies from a bottom-up and participatory approach, to articulate the different actors in the territories.

The first moment presents the generalities of the SDGs and their 2030 global agenda. A second section explains the PDTs within the framework of the Peace Agreement in Colombia. The third phase seeks to generate reflection on the harmonization between the 
SDGs and the PDET, recognizing points of encounter and differences as a strategy that promotes social sustainability. In the fourth section of this article, we will discuss the design of participatory public policies from a bottom-up approach that allows contributing to the construction of peace; since the PDET and the SDGs are designed in the means of participation to respond effectively to the needs of communities and their environment. Finally, we will present the conclusions that show how the PDET with the SDGs could articulate in scenarios of transformation and renewal of territories in Colombia.

Keywords: PDET, ODS, territory, colombian postconflict, social sustainability.

\section{INTRODUCCIÓN}

En el año 2015, 193 países a nivel mundial coincidieron en la necesidad de crear una nueva agenda mundial para el 2030 con una financiación integral para el desarrollo, en la que se adoptaron temas enfocados a la mitigación de los efectos del cambio climático y la promoción del desarrollo sostenible, dando así una agenda ambiciosa que contiene 17 Objetivos de Desarrollo Sostenible (ODS) y 169 metas que buscan erradicar la pobreza, combatir la desigualdad y promover la prosperidad, bajo un enfoque diferencial y territorial. Los ODS se reconocen como un llamado universal a proteger el planeta y a garantizar que todas las personas gocen de paz y bienestar. Así, alrededor del mundo, han surgido diferentes experiencias e iniciativas de diversas escalas que buscan la integración y la armonización a la Agenda generada por los ODS.

Para el caso colombiano, una de las apuestas orientadas a la articulación de la agenda nacional de los ODS se presenta en el marco del Acuerdo de Paz que se consolidó entre el gobierno del expresidente Juan Manuel Santos y la extinta guerrilla de las FARC-EP. Dentro de este proceso en específico existe una estrategia que pretende aportar a la implementación de los acuerdos. Esa es conocida como "los Programas de Desarrollo con Enfoque Territorial" -PDET-. Dichos PDET son programas subregionales que se consolidaron con el fin de reparar a los municipios más afectados por el conflicto armado desde un enfoque territorial que responda a las 
necesidades específicas del territorio y sus comunidades. Por esto, se han generado esfuerzos que buscan transformar los espacios de violencia por zonas de paz y de reparación.

De igual forma, en el acuerdo firmado se evidencia una apuesta de cambio en los territorios y el desarrollo de estos. Un ejemplo de esto es la formulación de diferentes herramientas que permiten la restauración de estos espacios por medio de diferentes puntos planteados en la Agenda de paz, como el Punto 1. Hacia un nuevo campo colombiano: Reforma rural integral, el Punto 4. Solución al problema de las drogas ilícitas, y no menos importante el Punto 5. Sistema Integral de Verdad, Justicia, Reparación y No Repetición. En la interpretación de cada tema se puede identificar la vinculación que se tiene para un enfoque territorial y una transformación de los territorios, lo que permite una posible articulación con los Objetivos de Desarrollo Sostenible -ODS- busquen propiciar sociedades más sostenibles y en paz, dado que "los ODS contienen medidas audaces y transformadoras para consolidar el camino de la sostenibilidad de manera que se incluyan a diferentes segmentos de la población" (DNP, s.f., p. 1).

Se debe entender que, en los ODS, la sostenibilidad es el punto clave para el cumplimiento de las 169 metas debido a que hoy en día esta representa un nuevo paradigma de las agendas públicas y del de desarrollo social, cuyo origen se remonta a los años ochenta y surgió como iniciativa del informe Brundtland, el cual pone en discusión la posible armonización entre desarrollo y sostenibilidad, para luego enfocar su definición de desarrollo sostenible hacia la "conjunción del derecho al desarrollo con el aprovechamiento respetuoso de los recursos naturales" (Aguado, 2018, p. 20).

Por lo anterior, es importante comprender que en los procesos que lleven a cabo el desarrollo de las experiencias que buscan implementar los ODS haya un proceso activo de participación por parte de las comunidades, capaz de orientar las propuestas a la construcción y fortalecimiento de la sostenibilidad social, es decir, lo que aporta en la relación de un territorio y sus habitantes.

La metodología propuesta en el presente artículo parte de un enfoque cuantitativo, basado en la exploración de diferentes fuentes bibliográficas y datos descriptivos que permitan entender y analizar la posible armonización entre los Planes de Desarrollo con Enfoque 
Territorial -PDET- y los Objetivos de Desarrollo Sostenible -ODS-. Esta posible vinculación se realizará por medio de técnicas metodológicas como la revisión documental, la recopilación de datos existentes -como otros instrumentos territoriales que se han puesto en marcha para mejorar las condiciones de los territorios y las comunidades-, las estadísticas, que permiten visibilizar el compromiso existente de las instituciones a cargo y el análisis de datos de fuentes secundarias que puedan dar una argumentación sólida respecto a la posible relación entre los ODS y los PDET.

\section{Objetivos de Desarrollo Sostenible -ODS-}

Los Objetivos de Desarrollo Sostenible -ODS- surgen en la segunda década del presente siglo como apuesta de los Estados miembros de las Naciones Unidas para poder mitigar problemas mundiales como: la pobreza, el medio ambiente y la calidad de vida de todos los habitantes del planeta, construyendo así una agenda global 2030. La agenda se compone de 17 ODS proyectados a 15 años. Estos objetivos responden a algunas necesidades del ser humano y de la naturaleza, entre los que se destaca el hambre cero, agua limpia y saneamiento, igualdad de género y garantizar la vida de los ecosistemas terrestres, entre otros. En los cuales se busca un desarrollo sostenible desde todos los pilares propuestos por las Naciones Unidas. De ahí deriva, por tanto, la opción de mejorar las condiciones de vida del mundo poniendo en práctica los ODS, partiendo de la premisa de que el medio ambiente, la sociedad y la economía, deben interactuar conjuntamente para un desarrollo óptimo de la convivencia y respeto por nuestro ecosistema (Perea, 2019, p. 123).

El desarrollo sostenible se ha entendido como la capacidad de "satisfacer necesidades del presente sin comprometer las necesidades de las futuras generaciones" (Brundtland, 2019, en Perea 2019, p. 123) comprometiéndose así con el futuro de la comunidad, la cual permite la concentración de esfuerzos para construir un futuro inclusivo y resiliente para el planeta y las personas.

Los 17 ODS buscan una armonización entre tres elementos básicos: el crecimiento económico, la inclusión social y la protección del medio ambiente, todos esenciales para el bienestar social, personal y natural de los entornos naturales. Sin embargo, este compromiso 
no solo se puede entender desde el ejercicio exclusivamente gubernamental, sino que por el contrario "debe haber una coordinación entre políticas públicas, empresas, sector privado, sociedad civil y academia, debido a que conjuntamente se debe afrontar el reto y las actividades deben de ser puestas en práctica por todos" (Perea, 2019, p. 124).

Estos objetivos se rigen bajo cinco lineamientos mundiales que se conocen como las $5 P$, por su nombre en inglés: 1 ) personas (people), con esto se busca la dignidad de igualdad de todas las personas en un espacio saludable; 2) planeta (planet), enfocar todos los esfuerzos en poner el ecosistema como punto central de la problemática mundial; 3) prosperidad (prosperity), poder transformar las economías de los países para así vivir en armonía con la naturaleza; 4) paz (peace), poder propiciar comunidades pacíficas en cada espacio; y 5) alianzas (partnerships), construir acuerdos y pactos mundiales entre los diferentes actores por medio de los ODS.

Los ODS se aplican mediante la Agenda de acción de Addis Adeba (2015), que fue adoptada por la Tercera Conferencia Internacional sobre Financiación para el Desarrollo, llevada a cabo en julio de 2015. Esta agenda expresa un acuerdo mundial enfocado a la financiación del desarrollo sostenible, ya sea con recursos públicos y privados, nacionales o internacionales. Dicho acuerdo se articula en siete esferas intersectoriales: a) prestación de protección social y servicios públicos para todos; b) amplio esfuerzo para poner fin al hambre y la malnutrición; c) superar las carencias en materia de infraestructura; d) industrialización inclusiva y sostenible; e) generación de empleo productivo y trabajo decente para las micro, pequeñas y medianas empresas; d) protección de los ecosistemas; y g) promoción de sociedades inclusivas y pacíficas. De igual manera, la Agenda de financiamiento se ocupa de diferentes componentes económicos, como cooperación internacional para el desarrollo, la sostenibilidad del endeudamiento externo, los sistemas tributarios, la ciencia, tecnología, las innovaciones y el comercio, entre otras. Esto, con la finalidad de la movilización de los recursos financieros para la apropiación y el cumplimiento de los 17 ODS y de las 169 metas.

La supervisión de los ODS se realizará por medio de indicadores mundiales que ha desarrollado el Grupo Interinstitucional de expertos. Se igual forma, los gobiernos territoriales también elaborarán 
objetivos nacionales para contribuir al seguimiento de los progresos conseguidos en el marco de los ODS.

Por su parte, un año después de la Agenda 2030 se firmó en Colombia (2016) el Acuerdo de Paz, que abría la posibilidad de un nuevo camino nacional para intentar poner fin a la dilatada situación de violencia y de terror por más de seis décadas. La implementación se realiza en diferentes dimensiones y etapas, buscando restablecer los derechos de las víctimas y sus territorios, lo que permite el reconocimiento de todas aquellas personas que fueron vulneradas por la guerra, para poder buscar una solución equilibrada entre los negociadores, los acuerdos estipulados y su implementación. Justamente, por este enfoque, y por la propia naturaleza del conflicto, los acuerdos de paz dan paso a la consolidación de una estrategia territorial, la cual busca promover el desarrollo de las zonas rurales y el crecimiento económico de las mismas, y para ello se diseñaron los Programas de Desarrollo con Enfoque Territorial -PDET-.

\section{Programas de Desarrollo con Enfoque TerRItORIAL -PDET-}

El primer punto del Acuerdo Final de Paz, "Reforma rural integral", establece una reparación colectiva desde un enfoque territorial, que se materializa en los Planes de Desarrollo con Enfoque Territorial -PDET-, los cuales tienen por objetivo "lograr la transformación estructural del campo y el ámbito rural y un relacionamiento equitativo entre el campo y la ciudad" (Acuerdo Final, 2016, p. 21).

Los Planes de Desarrollo con Enfoque Territorial -PDET- buscan mejorar la calidad de vida de los habitantes de los territorios afectados por el conflicto armado a partir de 8 pilares: 1) ordenamiento social de la propiedad rural y uso del suelo; 2) infraestructura y adecuación de tierras; 3) salud rural; 4) educación rural y primera infancia; 5) vivienda, agua potable y saneamiento básico rural; 6) reactivación económica y producción agropecuaria; 7) sistema para la garantía progresiva del derecho a la alimentación; y 8) reconciliación, convivencia y construcción de paz (Confianza y Paz, s.f.).

Estos programas se construyeron a partir de tres fases: la fase veredal, en la que se aprueba el Pacto Comunitario para la Transformacional 
Regional (PCTR); la fase municipal, en la cual se toma como insumo el PCTR y se incluyen nuevos actores; por ejemplo, el sector privado, organizaciones sociales, entre otros, y se construye el Pacto Municipal; y la fase subregional, en la que los delegados de los municipios aprueban el Plan de Acción para la Transformación Regional (PATR) de cada una de las 16 subregiones PDET y los 170 municipios priorizados (Ley 893, 2017).

Para ser eficaces, los PDET se instrumentalizan a partir de los Planes de Acción para la Transformación Regional (PATR), los cuales se construyeron mediante pactos comunitarios, étnicos y municipales, definiendo así las apuestas del territorio para el desarrollo integral. Estos planes, deben materializar la planeación a 10 años y se deben revisar y actualizar cada 5 años en las regiones. De esta manera, el Gobierno ha institucionalizado los PATR en 16 subregiones y 170 municipios, en donde se realizó un proceso de planeación participativa con más de 6,6 millones de habitantes que habitan en estos territorios vulnerados.

Estos Planes de acción deben contemplar, además del enfoque territorial, un enfoque diferencial que priorice los grupos más vulnerables. Uno de los aspectos más importantes de la construcción e implementación de los PDET es el diálogo constante con las comunidades de los territorios, ya que este enfoque participativo permite localizar e identificar más eficientemente las necesidades, demandas y prioridades de las personas que habitan las zonas priorizadas y que sean ellas mismas quienes propongan soluciones a estos problemas.

\section{Armonización entre ODS y PDET}

Abordamos ahora el tema de este artículo: la , posible armonización entre los ODS y los PDET, buscando así un acercamiento entre la sostenibilidad social, la calidad de vida de los habitantes, el medio ambiente y en este caso, de los municipios PDET.

Las variables seleccionadas surgen e la preocupación de indagar acerca de la importancia que tienen los programas subregionales PDET en un escenario de posconflicto debido a que estos nacen después de un largo proceso de concertación entre la guerrilla de las FARC y el 
Estado colombiano, preparando así los territorios como espacios de reparación y diálogo permanente para las víctimas.

Es por esto, que los PDET se suman a la iniciativa de la Agenda 2030 con la apuesta de los nuevos -ODS-, lo que permite entender un panorama a nivel global desde un enfoque particular y específico como lo son los programas PDET. Desde esta mirada, se evidencia la transformación a la cual se apuesta por medio de las dos herramientas, en donde surgen necesidades puntuales que se buscan erradicar o mitigar por medio de un cambio económico, social y ambiental que posibilite garantizar la calidad de vida de las personas y la protección del planeta.

La calidad de vida introduce los aspectos ambientales en intersección con las necesidades humanas. Elmedio Ambiente (...) es el campo de acción para una calidad de vida en la ciudad, implicando mo sólo la aplicación de los principios de sostenibilidad ecológica. (Hernández, 2009, p. 8)

Los PDET y los ODS buscan lo mismo: cumplir a la sociedad y al medio ambiente, donde se convierten en estrategias claves para poder alcanzar una sostenibilidad social, la cual permite un enlace entre tres esferas: la naturaleza, la sociedad y las instituciones, que median la relación entre las dos primeras. Esto evidencia que, tanto los PDET y los ODS son manifestaciones territoriales, sociales, ambientales y económicas que pretenden generar renovación y evolución en los municipios PDET y en las naciones a nivel mundial.

Por ello, los s ODS se consideran un llamado universal a los diferentes países para que puedan poner fin a necesidades puntuales que se generan a nivel mundial y que llevan a que la población conviva con la pobreza, el hambre, la baja educación y el cambio climático, entre otros. Es así, que en el 2015 se estipularon los 17 ODS que dan respuesta y que ayudan a eliminar dichas carencias que se presentan en el mundo. Estas problemáticas, no solo se han planteado desde instituciones como las Naciones Unidas o programas como el PNUD, sino que por el contrario se han evidenciado apuestas desde territorios y países que le apuntan a la realización de varios ODS. 
Como se mencionó anteriormente, Colombia le ha apostada a una transformación de los territorios después de la firma del Acuerdo de paz, donde surgieron diversos puntos que ayudan a mitigar las brechas existentes entre la ciudad y el campo, permitiendo así que los PDET se puedan convertir en una estrategia local que trabaje en la promoción de algunos OSA; sobre todo, teniendo en cuenta que en el año 2020 inició la fase de "La Década de Acción la cual exige acelerar soluciones sostenibles dirigidas a los principales desafíos del mundo. Desde la pobreza y la igualdad de género, hasta el cambio climático, la desigualdad y el cierre de la brecha financiera" (Naciones Unidas, s.f.). Esto, uniendo diversos esfuerzos no solo políticos entre naciones, sino de diversas esferas a través de las comunidades de los países involucrados.

A 9 años de que se cumpla la fecha, se están aunando esfuerzos en todo el mundo para cumplir con la promesa de la Agenda 2030, mediante la movilización de más gobiernos y empresas, así como con un llamamiento a todas las personas para que hagan suyos los Objetivos Mundiales (Naciones Unidas, s.f.).

Por lo cual, el secretario general de la ONU en el 2019 realizó un llamado a los diferentes sectores de la sociedad para que trabajaran desde sus posibilidades por los ODS, planteando tres esferas de acción: 1) acciones a nivel mundial; 2) acciones a nivel local: y 3) acciones por parte de las personas. Es importante recalcar la necesidad de que en estos componentes existan procesos de participación activa, como se expondrá más adelante, lo que permite la vinculación entre diferentes agentes sociales para trabajar por la transformación y sostenibilidad social. Así mismo, plantean 3 factores importantes para la efectividad de esta Década de Acción: 1) movilizar a todas las personas en el mundo, 2) exigir urgencia y ambición, y 3) poder impulsar ideas para que se conviertan en soluciones (Naciones Unidas, s.f.).

Este último componente, tiene relación con la materialización de los PDET desde el territorio colombiano, debido a que pueden ser una apuesta para dar solución a las necesidades planteadas a nivel mundial, ya que por medio de un enfoque territorial y de una apuesta bottom-up se puede reparar a las víctimas afectadas por el conflicto armado interno, permitiendo así un desarrollo económico, social y ambiental de las comunidades, siendo estos los enfoques principales de los ODS con la Agenda 2030. Los PDET se pueden 
entender como pequeños OSA debido a que le apuestan al bienestar rural, a la protección multicultural, al desarrollo económico y de integración de las regiones golpeadas por el conflicto; y al poder transformar el campo en escenario de reconciliación. De igual forma, dichos pilares también serán medidos por indicadores, los cuales determinarán el progreso de los territorios colombianos.

Poe ello, en el segundo semestre del 2020, la Agencia de Renovación del Territorio (ART) presentó una rendición de cuentas en donde se explicó, que uno de sus mayores esfuerzos fue "aportar al cumplimiento de los Objetivos de Desarrollo Sostenible (ODS). La Agencia asumió el reto de fortalecer las metas trazadas en ellos, para así articular la agenda pública e implementar proyectos en los ocho pilares temáticos de los PDET" (Semana Rural, 2021, p. 1).

De esta manera, parece claro que existe una relación indirecta entre los ODS y los PDET, los cuales pueden aportar al fortalecimiento de la sostenibilidad social del país, dando paso a la construcción de una agenda nacional que permita la vinculación de diferentes actores sociales. Un ejemplo de esto es desde los pilares 4) educación rural y primera infancia, 5) vivienda, agua potable y saneamiento básico rural; y 6) producción agropecuaria, de los PDET, en donde se hace la vinculación con los ODS 1) fin de la pobreza; 4) educación de calidad; 6) agua limpia y saneamiento; 8) trabajo decente y crecimiento económico.

Esta correlación permite articular la educación rural con la capacidad de que los países puedan potencializar la educación de calidad. De igual forma, garantiza la vivienda y el agua potable con plenitud de garantizar agua limpia y saneamiento en las naciones. Por último, tener la capacidad de una producción agropecuaria que proporcione un trabajo decente y así tener un crecimiento económico.

Estos pilares permiten una oportunidad de desarrollo, tanto en el campo como en de los diferentes países, ya que se respalda un incremento a nivel social, ambiental y económico que se busca en los territorios y en las comunidades. La iniciativa de generar educación, vivienda y producción ayuda a que las sociedades piensen y le apuesten a nuevos emprendimientos y formas de generar compromiso con el cambio que el mundo necesita, siempre y cuando respondan a los ODS y en el caso específico de Colombia a los PDET. 
De igual forma, los pilares 3) salud rural y 7) derecho a la alimentación de los PDET, permiten una vinculación directa con los ODS 2) hambre cero y 3) salud y bienestar, ya que se apuesta a mejorar la calidad de vida desde el cumplimiento básico de las necesidades de la población, como lo es una salud y una alimentación con garantías y de manera justa con las comunidades.

Consecuente con esto, el enfoque 1) ordenamiento social de la propiedad rural y uso del suelo; 2) infraestructura y adecuación de tierras, tienen concordancia con los ODS 9 (industria, innovación e infraestructura) y 10 (reducción de las desigualdades). Esta conexión permite un crecimiento económico en los territorios colombianos y en las naciones, ya que existen instrumentos territoriales, como el ordenamiento social que puede reducir las desigualdades por medio de una infraestructura adecuada y de innovación en cada sector de la nación.

Siguiendo este mismo análisis, el pilar número 8) reconciliación, convivencia y construcción de paz de los PDET, está directamente relacionado con el ODS 16 (paz, justicia e instituciones sólidas), debido a que los dos enfoques le apuesta a la construcción de sociedades en paz, justas y sólidas que permiten un crecimiento desde lo social, lo económico y lo ambiental, tanto a nivel nacional y mundial, dando paso así a reparar a las víctimas del conflicto nacional e internacional y buscando una dirección hacia el cumplimiento de la satisfacción de necesidades básicas.

Todo lo anterior, busca armonizar dos instrumentos pensados desde y para la sociedad, donde instituciones públicas, privadas, culturales, y sociales tengan injerencia en el hacer de estas metas y garantías en el cumplimiento de las mismas, con la finalidad de poder alcanzar el ODS 11 (ciudades y comunidades sostenibles), que permitan una mayor armonización con las comunidades desde lo ambiental, lo social y lo económico.

El ODS 17 (alianzas para lograr los objetivos) es el que permite abrir un escenario de una participación activa de diferentes estrategias que aporten al logro de la Agenda Mundial, como lo puede ser en este caso, los Programas de Desarrollo con Enfoque Territorial -PDET-.

Desde la APC, en Colombia para el año 2019 se distribuyeron recursos de la siguiente manera: 
El 82,3\% de los recursos de cooperación internacional no reembolsable se dirigieron a cinco ODS: ODS 10 (reducción de las desigualdades), 39,33\%; ODS 16 (paz, justicia e instituciones sólidas), 16,52\%; ODS 11 (ciudades y comunidades sostenibles), 14,70\%; ODS 2, (hambre cero), 8,05\%; y ODS 8 (trabajo decente y crecimiento económico), 3,80\%. (APC Colombia, 2020, p. 22)

Lo anterior muestra que es posible contar con recursos para la aplicación de los ODS en el país, y claramente apoyando los que se relacionan con los PDET, ya que muchas de las metas que se encuentran relacionadas a los 5 ODS financiados en el país tienen relaciones directas con las metas del Acuerdo Final de Paz, entre las cuales podemos encontrar, la reincorporación económica de excombatientes de las FARC, el fin del conflicto, y el desarrollo de los territorios más afectados por la violencia, por lo que los ODS número 10 y 16 encabezan la lista de recursos de cooperación internacional (APC Colombia, 2020).

Los PDET no solo permiten mejorar las condiciones de vida de los habitantes de los 170 municipios priorizados en el país por sus afectaciones en el marco del conflicto armado, sino que además son una de las estrategias más notables en el país para aportar en la promoción de los ODS, permitiendo que se construya un Estado que sea guía para las diferentes naciones que han sufrido de dinámicas de violencia. De igual forma, la propuesta de una posible armonización entre los ODS y los PDET permiten la construcción de un camino hacia la sostenibilidad social, la cual permite un desarrollo con el entorno y la consolidación de un progreso ambiental, económico y social, en donde se evidencia el alcance de indicadores logrados por las naciones.

\section{Políticas públicas participativas}

Entre los diferentes puntos en los que podrían coincidir los ODS y los PDET está la necesidad de ser construidos o reconfigurados a partir de la participación comunitaria. Esto, debido a que son precisamente ellos, y ellas, los beneficiarios de estas políticas y quienes de manera directa se ven afectados por el desarrollo de las mismas, además de conocer de primera mano los territorios en los cuales se 
implementarán, por lo que sus aportes son vitales para la comprensión de los impactos reales de las políticas públicas.

Por lo anterior, este apartado se acerca a los conceptos de participación comunitaria o ciudadana y al enfoque bottom-up como dos formas de comprender la importancia y necesidad que existe de que los procesos encaminados a la construcción de paz territorial, a la toma de decisiones de interés colectivo y a la transformación de los territorios sea dada de forma participativa y, sobre todo, desde aquellas personas que conforman los diferentes territorios del país.

Para el caso del PDET, aunque se consolidó su forma como programa en la Habana, se buscaba que su desarrollo se hiciera de forma participativa debido a que "la participación activa de las comunidades - hombres y mujeres - en conjuntos con las territoriales, es la base de los PDET" (Acuerdo Final, 2016, p. 22). Así, desde el inicio de su implementación, estos programas fueron diseñados de forma comunitaria, iniciando en un nivel veredal (teniendo en cuenta que en su mayor porcentaje se desarrollan en zonas rurales), luego el nivel municipal (bajo la pregunta de ¿cómo imaginamos nuestros territorios?) y, finalmente, el nivel subregional. Estas tres fases contaron siempre con la participación activa de las comunidades, que desde su conocimiento y experiencia proponían ideas y soluciones alrededor de las dinámicas y dificultades de su territorio, acompañados de las instituciones encargadas de llevar a cabo los encuentros y cumplimiento de las acciones concretadas.

El resultado del trabajo anterior fueron 16 planes de acción PDET que se construyeron con la participación de más de 200000 personas, de las cuales el $25 \%$ corresponde a grupos étnicos y el $41 \%$ son mujeres, proponiendo 32808 iniciativas territoriales (Agencia de Renovación del Territorio, 2021). Estos 16 planes corresponden a las 16 subregiones PDET existentes en el país, por lo que se diseñó un plan diferente para cada una, entendiendo que estos programas están estructurados en clave territorial haciendo necesario ajustar acciones a cada territorio, reconociendo la diversidad de sus comunidades, dinámicas y características físicas específicas de cada zona, posibilitando la creación de:

Un diagnóstico objetivo, elaborado con la participación de las comunidades — hombres y mujeres—, en el que se consideren bajo el 
enfoque territorial señalado las necesidades en el territorio y las acciones que coordinen los diferentes elementos, y tenga metas claras y precisas que posibiliten la transformación estructural de las condiciones de vida y de producción. (Acuerdo Final, 2016, p. 22)

Por lo anterior, con el fin de proporcionar metas claras y precisas se consolidó la hoja de ruta en el Plan de Desarrollo Nacional 20182022 "Pacto por Colombia - Pacto por la Equidad", que establece la forma en la que estos 16 planes de acción se desarrollarán en un periodo de 15 años, manteniendo siempre la participación comunitaria para decidir acerca de las estrategias para la transformación de su territorio en los planos ambientales, económicos y sociales. A estas estrategias se le conocen como escenarios meta (Agencia de Renovación del Territorio, s.f., p. 1).

Así, la hoja de ruta funciona como uno de los mecanismos de participación para los PDET y esta cuenta con 3 momentos generales: 1) la construcción técnica de la hoja de ruta: en la cual un equipo especializado genera un contexto de las subregiones con datos y fuentes oficiales; 2) la socialización de la metodología de la hoja de ruta: donde los y las participantes de las comunidades y sus delegados discuten alrededor del resultado del primer paso y brindan nuevas características del territorio y sus dinámicas desde sus propias experiencias y conocimientos; $y$, finalmente, 3) la construcción participativa: que permite la validación territorial de la hoja de ruta, en la cual se genera un espacio de discusión y decisión para definir los momentos y montos económicos que llevará a cabo la hoja de ruta para la transformación del territorio. En este punto, la participación de las comunidades y sus delegados es vital, pues asumen su agencia como sujetos sociales para incidir en la gestión pública.

Algo importante de esta hoja de ruta es que articula diferentes planes nacionales y territoriales de los 170 municipios PDET, tales como: Planes de la Reforma Rural Integral, Planes Integrales de Reparación colectiva, Planes Integrales de Sustitución y Desarrollo Alternativo, Planes Estratégicos de Intervención Integral y Planes de Desarrollo; ya que "es necesario elaborar de manera participativa un plan de acción para la transformación regional, que incluya todos los niveles del ordenamiento territorial, concertado con las autoridades locales y las comunidades" (Acuerdo Final, 2016, p. 22). Esto implica también la importancia de armonizar políticas 
públicas que puedan apoyarse entre sí, y no, que en su aplicación sean contradictorias.

Se hace además necesario que la participación comunitaria sea activa, no sólo en la fase de la planificación sino también en todos sus componentes, desde el desarrollo hasta su seguimiento y evaluación. Un ejemplo en los PDET de esto son las "obras PDET" las cuales son acciones de mejoramiento físico de las zonas, en las que las comunidades son quienes planean las obras que deben llevarse a cabo y ordenarlas en prioridad, asumen desde juntas de acción comunal el desarrollo de las obras y, finalmente, acompañan el proceso de construcción y evaluación de la obra (Agencia de Renovación del Territorio, 2020).

Sin embargo, para que la participación de las diferentes comunidades sea activa, no solo basta con disponer los espacios, sino también se deben ofrecer las garantías necesarias, desde una visión territorial, ya que:

El enfoque territorial de las comunidades rurales que tenga en cuenta las características sociohistóricas, culturales, ambientales y productivas de los territorios y sus habitantes, así como sus necesidades diferenciadas en razón de su pertenencia a grupos en condiciones de vulnerabilidad. (Acuerdo Final, 2016, p. 22)

Reconociendo así las medidas necesarias de seguridad y todos los enfoques diferenciales que proporcionen garantías para el ejercicio de la participación comunitaria, y la construcción de un proceso participativo fuerte que promueva la paz estable y duradera en estos territorios afectados por el conflicto armado interno. Teniendo en cuenta que esta última condición ha marginalizado a diferentes movimientos sociales, o tipos de organización social, y es obligación del gobierno velar por su seguridad y participación para que se sumen a la apropiación de las discusiones y decisiones sobre su territorio, debido a que:

[...]posibilita construir ciudadanías críticas con un sentido profundo de sus deberes frente al cuidado de lo público, además de contribuir con la evaluación crítica de la gestión pública y la construcción de nuevas éticas sobre el rol del Estado y la sociedad. (Ligarreto, 2017, p. 244) 
Estas relaciones posibilitan la reconfiguración de lo público e incidir en su gestión mientras se mantenga una participación activa entre las comunidades y la institucionalidad, factores clave para la sostenibilidad social, y aunque en el Acuerdo Final (2016):

[...]se garantizará la participación plena y efectiva de los representantes de las autoridades étnicas y sus organizaciones representativas en las diferentes instancias que se creen en el marco de la implementación del Acuerdo Final, en particular las consagradas en el Punto 2 y las instancias de planeación participativa. (p. 206)

También se menciona que:

En ningún caso pretenden limitar las competencias de ejecución de los gobernantes, ni las competencias de órganos colegiados (Congreso, concejos y asambleas). En el marco de los PDET se establecerán expresamente las características generales y tiempos para garantizar el buen funcionamiento de estos mecanismos de participación. (Acuerdo Final, 2016, p. 23)

Por lo que es necesario que se amplíe la capacidad de acción de participación de las comunidades, posicionándose al mismo nivel de las instituciones y no limitando su ejercicio a simples socializaciones de resultados, además de ser vital la voluntad política para que esto se logre. Propiciando que "los Planes de Desarrollo con Enfoque Territorial (PDET); que desde su definición implican alance político en tanto reconfiguran lo público, permiten la incidencia en la gestión pública y fortalece el Estado social de derecho" (Ligarreto, 2017, p. 234), involucrando como se ha mencionado anteriormente en todos las fases del programa a la comunidad e impactando de maneras notables.

Para conseguir lo anterior, un aporte significativo podría darse desde la planeación del enfoque de bottom-up "este enfoque tiene como objetivo que se desarrollen y articulen medidas cooperativas entre autoridades y comunidades, pensándose las características y necesidades de cada territorio" (Ramírez, 2021) ya que "se trata de hacer valer los derechos y las capacidades de la gente, que sientan como propio el esfuerzo de reconstrucción" (Jaramillo, 2018, p. 4). 
El diseño participativo con un enfoque bottom-up apunta a la reconstrucción desde las unidades microsociales, las más pobres y vulnerables, las periferias. Esta reconstrucción desde abajo deberá ser material y moral a la vez, pues si bien implica contar con las bases materiales para salir adelante - por ejemplo, contar con una vivienda digna-, también requiere de la reconstrucción de una ética de la vida cotidiana, la cual se apoye en redes colaborativas, familiares y barriales que hagan posible nuevos horizontes. (Lemaitre, 2018, en Ramírez, 2021)

Precisamente, estos procesos de participación mancomunada y desde abajo son los que pueden fortalecer la participación local y la gestión de lo social en lo público, abriendo espacios de discusión y decisión para las comunidades que históricamente han sido marginalizadas e invisibilizadas en planos políticos, y quienes desde sus experiencias pueden aportar a la apropiación de las soluciones que necesitan en sus territorios, ya que hacen parte de la realidad de su propio vivir.

El enfoque de bottom-up en la planeación de políticas públicas tiene una relación estrecha con los procesos de construcción de paz y posconflicto, pues esto se hace evidente en la revisión bibliográfica realizada y en diferentes casos del país en los cuales, por ejemplo, las víctimas, son centrales para la toma de decisiones y planeaciones de las políticas, donde se tienen en cuenta "on everyday events and the inclusion of local agencies in peacebuilding processes for varieties of peace" (Le Billon et al 2020, p. 4); en nuestro caso, específicamente la paz territorial que incluyendo a los diferentes actores locales de un territorio busca promover soluciones más efectivas, pues aunque "as such, it is not only a peace for the territory, but also from the territory" (Le Billon et al 2020, p. 4).

La paz territorial además se puede presentar "as a supposedly new vision and set of practices to build peace througha bottom-up territorialisation process" (Le Billon et al 2020, p. 20). Lo que hace evidente que, en los procesos relacionados a la construcción de paz en el país, es importante consolidar una mayor participación de los actores locales de los territorios para poder avanzar en las medidas que se buscan aplicar. De lo contrario, existirá un proceso más lento y con muchos retos que no permitan desarrollar desde las instituciones sus propósitos en los territorios. 
El enfoque bottom-up, o estas miradas de abajo hacia arriba, suponen una capacidad de todas y todos los actores locales que impulsen formas alternativas de construir paz. Esto quiere decir, iniciativas que se den desde el conocimiento propio de sus territorios, y no modelos que sean implantados desde otras latitudes (Mac Ginty, 2010, p. 403).

Roger Mac Ginty se ocupa de un concepto interesante, el de la paz cotidiana; concepto que reúne las diferentes accione que son creadas por las comunidades en sus zonas donde habitan para convivir en paz y construir proceso que se acerquen a esta convivencia desde prácticas locales, esto permite apropiarse del contexto de cada persona y legitimar de manera más fácil los proceso que se llevan a cabo.

Everyday peace has advantages that externally inspired and top-down peace initiatives may have difficulty in mobilizing. Primarily, everyday peace, given its bottom-up origins, may have a chance of legitimacy and authenticity that may be lacking from imported initiatives. Legitimacy and authenticity can only go so far, of course, especially if they have few material resources. Yet legitimacy and authenticity are often precisely what imported peace initiatives lack. (Mac Ginty, 2014, p. 561)

Esa paz cotidiana, o esa paz territorial, reafirma la necesidad de que sean las personas locales quienes aporten a la solución de sus necesidades puntuales, por lo que priorizan las condiciones en las que se encuentran y las habilidades o proyectos que necesitan para mejorarla. Esto último, es algo que en muchas ocasiones puede verse ignorado por los modelos importados que buscan implementarse en contextos diferentes, dificultando su eficacia. Además,

[...] incorporar a la ciudadanía en la construcción de escenarios futuros deseados, deliberados y consensuados colectivamente; a trabajar en la fase de diagnóstico de la formulación de los planes de desarrollo para identificar proyectos dinamizadores de inversión social que permitan construir bases sólidas para la paz y conseguir, en el mediano y largo plazo, cerrar la brecha de desigualdad existente entre el campo y la ciudad; en definitiva, planes que ayuden a dinamizar el desarrollo social, económico y productivo de los territorios. (Valencia, 2020, p. 7) 
Es por esto, que se hace necesario extender los mecanismos de participación de las políticas públicas y de los programas institucionales que aporten a la sostenibilidad social, lo que permite una mejor gobernanza y fomentando sociedades transparentes y en paz, donde los actores sociales sean tenidos en cuenta. Aunque los ODS no responden a un periodo de posconflicto, para el caso de los PDET este proceso de participación comunitaria se hace indispensable, ya que en experiencias internacionales la participación de las ciudadanías en estos escenarios es una herramienta de transformación y empoderamiento comunitario que puede reestructurar las relaciones de poder entre las comunidades y las instituciones (Lundy y McGovern, 2008).

\section{Conclusiones}

En suma, se evidencia una correlación entre los Objetivos de Desarrollo Sostenible -ODS- y los Programas de Desarrollo con Enfoque Territorial -PDET - debido a que son apuestas que permiten el mejoramiento tanto de las poblaciones como de los territorios, teniendo como ejes principales la calidad de vida, el medio ambiente, el crecimiento económico y las desigualdades (que en el caso de los PDET es tratar de mitigar las diferencias entre lo urbano y lo rural). Los ODS y los PDET se articulan en la medida del lenguaje transformador que contienen debido a que buscan el cambio desde miradas equilibradas y justas con los territorios y sus habitantes, lo cual permite la injerencia de diferentes actores sociales que ayudan al cumplimiento de las metas acordadas.

Por esto, la participación comunitaria en estos procesos es vital, ya que la sostenibilidad social también se fortalecerá en la medida en que los diferentes actores de un territorio se involucren entre sí para poder aportar soluciones a las necesidades que los aquejan, ayudando no solo al territorio en el que se encuentran sino, además, de forma directa a las relaciones que se dan entre las comunidades y las instituciones, importante para el cumplimiento de lo acordado y de procesos transparentes que permitan la discusión y decisión de todas y todos los involucrados en su entorno social y natural.

Las vinculaciones dadas en este texto acerca de la posible relación entre los ODS y los PDET debe trascender los planos teóricos 
y políticos para poder desarrollarse de forma real e impactar de manera positiva en las comunidades y territorios en los cuales se implementen. Cada estrategia deberá contar con las participaciones e indicadores necesarios para medir la efectividad del proceso y la orientación necesaria que debe tener.

La necesidad de que estos procesos de construcción de paz, o de implementación de políticas públicas que buscan mitigar problemas sociales, sean gestados desde la participación colectiva es vital, además de evidente, pues el fortalecimiento de las comunidades y la apropiación territorial de su contexto permitirá proponer y desarrollar planes de acción centrados en cada zona que permitan de forma eficaz la solución de las problemáticas. La agencia de los actores locales evitará la imposición de modelos importados y motivará la creación de metodologías particulares que respondan a las necesidades propias del país.

El aporte del PDET no solo se evidencia en la posibilidad de que su implementación construya y fortalezca los procesos de paz en el territorio colombiano, sino que ponga a Colombia en el plano internacional desde la posibilidad de liderar procesos que promuevan los ODS, para el bienestar del país y el mundo. De esta manera, las dos herramientas aquejan un llamado a las sociedades, en donde se respete y se consolide procesos de trabajo que ayuden al cumplimiento de las diferentes fases tanto de los PDET como de los ODS, para así alcanzar la visión que se tiene propuesta para el 2030 y poder seguir construyendo municipios PDET a largo plazo.

\section{Referencias}

Acuerdo Final. (2016, 24 de agosto). Acuerdo Final, Integral y Definitivo para la Terminación del Conflicto y la Construcción de una Paz Estable y Duradera. https: / / bit.ly/36Jp3QB

Agencia Presidencial de Cooperación Internacional. (2020). Análisis del comportamiento de la cooperación internacional no reembolsable recibida por Colombia en el año 2019. https: / / bit.ly/3Ba0b2x

Agencia para la Renovación del Territorio. (2019). ABC PDET. Colombia.

Agencia de Renovación del Territorio. (2020). Obras PDET. Colombia. https:/ / bit. ly / 2UlbeVV

Agencia de Renovación del Territorio. (s.f.). Hoja de Ruta. Colombia. https://bit. ly / $3 \mathrm{hISbOb}$ 


\section{ANÁLISIS \\ JURÍDICO-POLÍTICO}

Aguado, A. (2018). El desarrollo sostenible: 30 años de evolución desde el informe Brundtland [tesis doctoral, universidad de Sevilla]. Repositorio Universidad de Sevilla. https: / / bit.ly / 3ihIrcR

Confianza y Paz. (s.f.). Pilares PDET. Colombia. https: / / confianzaypaz.com/pdet /

DNP. (s.f.). Objetivos de desarrollo sostenible- ODS. https: / / bit.ly/3rg7lxw

Gobierno de Colombia. (2017, 28 de mayo). Decreto 893 de 2017. https:/ / bit.ly/3r$\mathrm{fDqFO}$

Jaramillo, S. (2018). La Paz Territorial. Colombia. https: / bit.ly / 3eteoho

Le Billon, Philippe, Roa-García, María Cecilia y López-Granada Angelica Rocío (2020) Territorial peace and gold mining in Colombia: local peacebuilding, bottom-up development and the defence of territories. Conflict, Security $\mathcal{E}$ Development, 20(3):303-333. https: / / doi.org/10.1080 / 14678802.2020.1741937

Ligarreto, L. (2018). Participación social en el acuerdo final para la construcción de una paz estable y duradera. [tesis de maestría, Universidad Nacional de Colombia]. Repositorio institucional UN. https:/ / bit.ly/3eqsOia

Lundy, Patricia y McGovern, Mark. (2008). Whose Justice? Rethinking Transitional

Lundy, Patricia y McGovern, Mark. (2008). Justice from the Bottom Up. Journal of Law and Society, 35(2), pp. 265-292. https://doi.org/10.1111/j.14676478.2008.00438.x

Hernández Aja, Agustín. (2009). Calidad de vida y medio ambiente urbano: indicadores locales de sostenibilidad y calidad de vida urbana. Revista INVI, 24(65), 79-111. https: / / dx.doi.org/10.4067/S0718-83582009000100003

Mac Ginty, R. (2014). Everyday peace: Bottom-up and local agency in conflict-affected societies. Security Dialogue, 45(6), 548-564. https://doi. org/10.1177/0967010614550899

Mac Ginty, R. (2010). Hybrid Peace: The Interaction Between Top-Down and Bottom-Up Peace. Security Dialogue, 41(4), 391-412. https://doi. org / 10.1177/0967010610374312

Naciones Unidas. (s.f.). Década de acción. Diez años para transformar el mundo. Portal Naciones Unidas. https:/ / bit.ly/3wMzLAC

Perea, L. (2019). Los objetivos de desarrollo sostenible y su inclusión en Colombia. Revista producción + limpia, 14(1), 122-127). https: / / bit.ly/3euoj6h

Ramírez, M. (2021). Hacia una participación ciudadana transformadora en Colombia. Análisis de los Programas de Desarrollo con Enfoque Territorial (PDET). Estudios Políticos, (61). https: / / doi.org/10.17533/udea.espo.n61a04

Semana Rural. (2021). En 2020 implementaron 406 proyectos PDET: ART. Colombia. https:/ / bit.ly/3z5KtUj

Valencia, G. (2020). Planeación del desarrollo y construcción territorial de la paz. Estudios Políticos, (57):10-16. https:/ / dx.doi.org/10.17533/udea.espo.n57a01 